\title{
A Comprehensive Review on Bot - Discord Bot
}

\author{
Anirudh Verma ${ }^{1}$, Shashikant Tyagi ${ }^{1}$, Gauri Mathur ${ }^{2}$ \\ ${ }^{1}$ Computer Science and Engineering, Lovely Professional University, Jalandhar, Punjab, India \\ ${ }^{2}$ Assistant Professor, Computer Science and Engineering, Lovely Professional University, Jalandhar, Punjab, India
}

\section{Article Info}

Volume 7, Issue 2

Page Number: 532-536

\section{Publication Issue :}

March-April-2021

\section{Article History}

Accepted : 20 April 2021

Published : 28 April 2021

\section{ABSTRACT}

The bot's purpose is to make the Discord server a bit more fun by making it interactive and interesting. This bot brings together various people from all over the world and helps them remain connected to each other. Once people join the server they can use all the features of the bot and join voice channels to communicate or share ideas thoughts or just have a chat. This is a better version of WhatsApp, Microsoft teams and has surpassed both of them in the same field. Discord is a VoIP, instant messaging and digital distribution platform designed for creating communities. Users communicate with voice calls, video calls, text messaging, media and files in private chats or as part of communities called "servers". Servers are a collection of persistent chat rooms and voice chat channels. Discord runs on Windows, macOS, Android, iOS, Linux, and in web browsers.

Keywords :- Discord, Python, Reddit, MongoDB, Visualization

\section{INTRODUCTION}

Discord is growing in popularity. As such, automated processes, such as banning inappropriate users and reacting to user requests are vital for a community to thrive and grow. Automated programs that look and act like users and automatically respond to events and commands on Discord are called bot users. Discord bot users (or just bots) have nearly unlimited applications. For example, let's say you're managing a new Discord guild and a user joins for the very first time. Excited, you may personally reach out to that user and welcome them to your community. You might also tell them about your channels or ask them to introduce themselves. The user feels welcomed and enjoys the discussions that happen in your guild and they, in turn, invite friends. Over time, your community grows so big that it's no longer feasible to personally reach out to each new member, but you still want to send them something to recognize them as a new member of the guild. With a bot, it's possible to automatically react to the new member joining your guild. You can even customize its behavior based on context and control how it interacts with each new user.

Users right now can copy paste different images or videos but no feature of automatically getting these things on your text channel has been implemented yet. Discord offers connectivity with Spotify, YouTube, Steam etc but not with reddit. There is no program that helps send all of the news, polls, facts, images automatically to the different text channels. A System of added public messages which trigger upon

Copyright: @ the author(s), publisher and licensee Technoscience Academy. This is an open-access article distributed under the terms of the Creative Commons Attribution Non-Commercial License, which permits unrestricted non-commercial use, distribution, and reproduction in any medium, provided the original work is properly cited 
joining and leaving the server might help personalise the system. Discord as it is now offers not much features in its text channels. Bots are everywhere on discord but every bot on discord is made for a very specific reason such as just playing music from YouTube, Spotify or generating tokens, or an RPG text game.

In a world where communication is important to many people, It is important for platforms which offer the social and communication features to be interesting and interactive. Discord is a groupchatting platform originally built for gamers that has since become a general use platform for many kinds of communities. Discord is divided into servers, each of which has its own members, topics, rules, and channels. Discord also allows users to voice- and video-chat, as well as livestream games and other programs from their computers.

At a basic level, Discord is built to allow members to message each other. Each community is called a "server." If you're familiar with Slack, you can think of it as a less formal version of that app. Servers are filled with text channels (where you can type to talk to other people) and voice channels (where you can voice-chat with others). You can also share videos ,images, internet links, music, and more. Each server usually has multiple channels, each of which is dedicated to a different topic or has different rules. For example, you might have one channel to talk about a game, and another for general chatting, and another for pictures of cats. The possibilities are endless. Starting your own server is free, as is joining other servers. There are thousands of different Discord servers, each one dedicated to a different topic. If you have an interest in something, there's a good chance you can find a Discord server for it. This is especially true for video games, which make up the bulk of Discord's most popular servers.

\section{EXISTING SYSTEM}

Users in Discord are generally considered the base entity. Users can spawn across the entire platform, be members of guilds, participate in text and voice chat, and much more. Users are separated by a distinction of "bot" vs "normal." Although they are similar, bot users are automated users that are "owned" by another user. Unlike normal users, bot users do not have a limitation on the number of Guilds they can be a part of. The existing system of discord allows the usage of various bots for playing songs, mini games, text etc but no proper discord bot has been setup that adds extra customizability and ease of understanding towards the server.

Discord gives you the power to create your own place to belong. Your Discord server is your home, shared with only the special people you invite. Discord is a VoIP, instant messaging and digital distribution platform designed for creating communities. Users communicate with voice calls, video calls, text messaging, media and files in private chats or as part of communities called "servers". Servers are a collection of persistent chat rooms and voice chat channels. Discord runs on Windows, macOS, Android, iOS, Linux, and in web browsers. As of July 21, 2019, there are over 250 million users of this software. Currently Discord is one of the most used VoIP's in the world, the pandemic has only helped it become even more successful and it's ever-improving nature makes this platform unbeatable.

\section{Details of the system}

The bot's purpose is to make the Discord server a bit more fun by making it interactive and interesting. This bot brings together various people from all over the world and helps them remain connected to each other. Once people join the server they can use all the features of the bot and join voice channels to 
communicate or share ideas thoughts or just have a chat. This is a better version of WhatsApp, Microsoft teams and has surpassed both of them in the same field. Bot's plans to serve uses

1. Automate the interaction

2. Improve server response

3. Making bot interaction

4. Ease up new server joiners

\section{It's functions}

- Post text, images, and videos from various subreddits on Reddit. Actively read the message on the discord text channel

- Be able to identify commands and respond to them

- Assign names to members

- Be able to remind people about tasks on demand

- Create polls

- Respond to commands

Functional Requirements - The functional requirement for recommendation system

- Requirement in scope - The admin can remotely use the software to handle the any problems that arise during the working of bot.

- Requirement out scope - The bot responds and greets the new users, allows them to use all the features and also keeps posting new information in the text channels

- User Interface: Discord

- Description of the data to be entered into the Discord Bot

- Descriptions of operations performed by each trigger command

- Descriptions of work-flows performed by the bot

- Descriptions of system reports or other outputs
Non-Functional Requirements - This section of the Software Requirement Specifications gives a glimpse about the non-functional requirements needed for the proper functioning of the recommender system. It gives a glimpse of how the non-functional attributes influence the major software functions on duty and how they facilitate the data flow taking place among various participants on the system.

- Performance Requirement: Since we are aiming to make the system real-timed, the performance of making recommendations and updating these recommendations is a very important issue. To put this forward more clearly, the system processing speed should be fast enough so that the participants won't even realize that the processing of data is taking place. Moreover, our system should have the ability to handle multiple users at the same time.

- Safety: Not even a single safety requirement has been identified yet. As the project proceeds and in case we identify any safety issues, it would be instantly addressed and updated.

- Security: This is one of the most important nonfunctional attributes that must be taken care of. Since our system deals with a huge bulk of data, we need to make sure that its integrity is maintained throughout. Moreover, our dataset also contains personal information such as the user ID's, trigger command he/she has recently used etc. and hence security design must be precise so that data couldn't be altered or manipulated by any means until and unless there is a proper authentication and authorization to an individual to do so.

- Usability: The system will prove to have widespread scope and access. Many people can access at the same time

- Portability: This Discord bot will be deployable on any discord server. 


\section{Implementations}

The discord bot adds more functionality to the created server in the discord application. The server helps people gather into one place and facilitates a "Virtual Room" environment for people, this bot will communicate with everyone in the server using trigger commands. It will fetch responses from the database and its code to help with the same. The bot has many features and one of its key points is fetching data from reddit website. It can get you facts, news, posts and happenings all around the world and can also engage in fun activities like poll, nicknames and greetings. The bot communicates with the server then with the database and gets the desired result through the APIs as shown in the data flow diagram. It can be further added into many servers if necessary hence proving to be a ready-todeploy application after its creation and testing. The maintenance is flexible and easy for us though it does require the bot to be shut down for a while. it is possible to update the bot while its active which is a plus point.

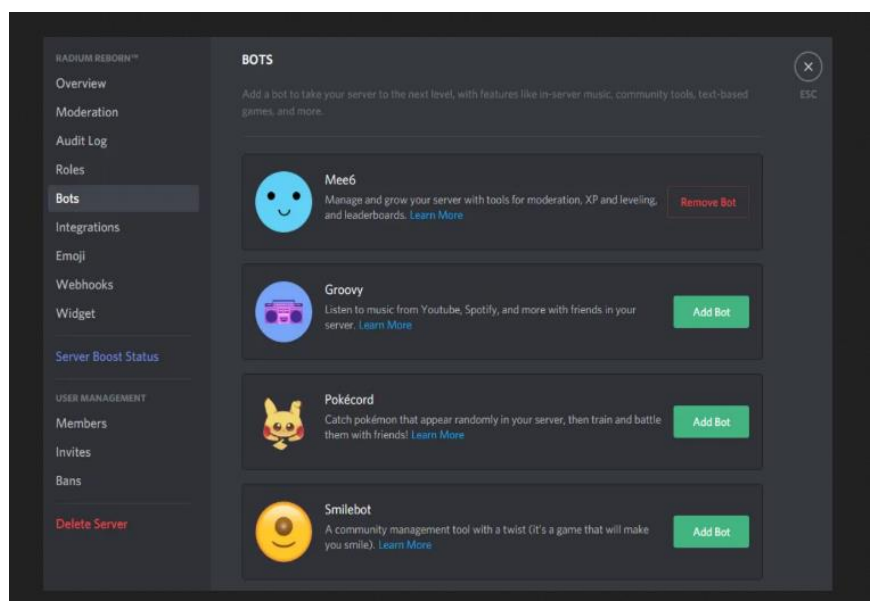

Figure 1 - Bot Created on Discord

\section{Comparisons}

Bot focuses on providing ease of usage as well as entertainment, unlike most other bots it is very easy to use and has the whole command list readily available. It is one of the few unique bots that fetch data from Reddit and provide the users in the server with it. It fetches data from the subbreddits via APIs and gives us updated and new results every $5 \mathrm{hrs}$ automatically. This bot is unique in purpose and has several functions as well. The bot provides us with functions such as viewing news, gifs, images and polls.It also does not read any personal information from the users present in the server and only gets activated when the trigger command is used. The bot also has some witty responses for the users just to make the platform more friendly and light hearted. It allows the users to manually select the channels and information they want. The Soviet Bot is deployable on any server and helps set itself up, moreover the bot doesnt require much hardware and is self sufficient for the most part. Even when updates are required it does not have to be shut down. The bot helps out with the general everday information and attempts to not bother via spam notifications. It basically is a cherry on top of a cake.

\section{Future Work}

Our discord bot also known as the Soviet bot is a universal tool that can be used by anyone that uses discord as a platform. It allows people to fetch data from reddit and other sites, provide them with information, entertainment and better accessibility. The bot can be deployed on any server and can setup itself as long as the admin gives it enough rights. The channels it sets up are all optional and can be selected or deselect as per choice. There are no restrictions on the number of servers it can join or on the number of people it can respond to. It can further in future be enhance for more activities and responses thereby allowing better personalisation for each individual server. The discord bots do not interfere with the regular working of a server and are secure. They increases interactivity and provide a better experience for all the users.Updates can be constant 
and will reflect on all the servers no heavy maintenance is required hence making it very robust. The discord bots can change the whole platform and can bring several enhancements while offering ease of use. They are viable and easy to maintain.

\section{REFERENCES}

[1]. Brian S. Butler. 2001. Membership size, communication activity, and sustainability: A resource-based model of online social structures. Information Systems Research 12, 4 (2001), 346--362.

DOI: http://dx.doi.org/10.1287/isre.12.4.346.9703

[2]. Eshwar Chandrasekharan, Chaitrali Gandhi, Matthew Wortley Mustelier, and Eric Gilbert. 2019. Crossmod: A Cross-Community Learning-based System to Assist Reddit Moderators. Proceedings of the ACM on Human-Computer Interaction 3, CSCW (Nov. 2019), 174:1--174:30. DOI: http://dx.doi.org/10.1145/3359276

[3]. R. Stuart Geiger. 2014. Bots, bespoke, code and the materiality of software platforms. Information, Communication \& Society 17, 3 (March 2014), 342--356. DOI: http://dx.doi.org/10.1080/1369118X.2013.87306 9

[4]. Charles Kiene, Andrés Monroy-Hernández, and Benjamin Mako Hill. 2016. Surviving an "Eternal September": How an online community managed a surge of newcomers. In Proceedings of the 2016 ACM Conference on Human Factors in Computing Systems (CHI '16). ACM, New York, NY, 1152--1156. DOI: http://dx.doi.org/10.1145/2858036.2858356

[5]. Wanda J. Orlikowski and Debra C. Gash. 1994. Technological frames: Making sense of information technology in organizations. ACM
Trans. Inf. Syst. 12, 2 (April 1994), 174--207. DOI: http://dx.doi.org/10.1145/196734.196745

[6]. Harmer, J. (2008). The Practice of English Language Teaching. Addison Wesley Publishing Company Essex

[7]. Lacher, Lisa. (2018). Using discord to understand and moderate collaboration and teamwork. Proceedings of the 49th ACM Technical Symposium on Computer Science Education, 1107- 1107

[8]. Pasupathi, Madhumathi 2013. Analyzing the effect of technology-based intervention in language laboratory to improve listening skills of first year engineering students. Profile, 15(1), 125-138

[9]. Zainol Abidin et al., (2011). Improving listening comprehension among malay preschool children using digital stories. International Journal of Humanities and Social Science, 1(14), 159-164

[10]. Yang et al., (2010). Integrating videocapture virtual reality technology into a physically interactive learning environment for English learning. Computers \& Education, 55(1), 1346 1356

\section{Cite this article as :}

Anirudh Verma, Shashikant Tyagi, Gauri Mathur, "A Comprehensive Review on Bot - Discord Bot", International Journal of Scientific Research in Computer Science, Engineering and Information Technology (IJSRCSEIT), ISSN : 2456-3307, Volume 7 Issue 2, pp. 532-536, March-April 2021. Available at doi : https://doi.org/10.32628/CSEIT2172100 Journal URL : https://ijsrcseit.com/CSEIT2172100 